\title{
Genetic Tabu search for robust fixed channel assignment under dynamic traffic data
}

\author{
Hakim Mabed • Alexandre Caminada • \\ Jin-Kao Hao
}

Received: 13 July 2009 / Published online: 24 November 2010

(C) Springer Science+Business Media, LLC 2010

\begin{abstract}
The contribution of this work is twofold. Firstly, we introduce a new channel assignment model for GSM radio networks. In this model both spatial and temporal variations of traffic are taken into account in order to improve network capacity and robustness. Secondly, using this model, we develop an original and effective hybrid algorithm to get high quality frequency plans. This algorithm combines a problem specific crossover and a Tabu search procedure. The proposed model and hybrid algorithm are evaluated using both artificial and real data. Computational results allow us to confirm the effectiveness of the proposed approach.
\end{abstract}

Keywords Hybrid genetic algorithms · Robust channel assignment · Traffic modeling $\cdot$ Real world OR application

\section{Introduction}

Over the past two decays, demand for mobile communication services has grown continually and rapidly. In such a context, it becomes more and more critical to be

A part of this work was carried out when the two first authors were with France Telecom R\&D.

H. Mabed

UFC, Laboratoire LIFC, 25200 Montbéliard, France

e-mail: hakim.mabed@pu-pm.univ-fcomte.fr

A. Caminada

UTBM, Laboratoire SET, 90010 Belfort cedex, France

e-mail: alexandre.caminada@utbm.fr

J.-K. Hao ( $\varangle)$

LERIA, Université d'Angers, 2 boulevard Lavoisier, 49045 Angers cedex 01, France

e-mail: jin-kao.hao@univ-angers.fr 
able to manage the system resources in a flexible manner [41]. In this paper, we consider the fixed frequency assignment problem where the traffic undergoes both spatial and temporal variations. Considering traffic variations for frequency assignment is desirable and even necessary to enable high network capacity and quality of service.

Many schemes of channel management have been adopted to cope with traffic variations [22]. Mainly, those schemes may be classified into three classes:

- Reactive schemes operate in real-time as new calls arrive in the system and consequently induce additional signaling loads in the network or require some changes to both base station and terminal equipment. Dynamic channel assignment [3-5, 7], hybrid channel assignment [38] and channel borrowing [40] belong to this class.

- Proactive or adaptive schemes periodically readjust the frequency plan according to expected traffic evolution [33]. This readjustment must be made in such a way that one performs a minimum number of modifications on the existing frequency plan in order to minimize channel handoff.

- Fixed schemes permanently allocate to each station a set of nominal channels. Frequency planning is then made on the basis of over-sizing of traffic data or using probabilistic distribution of traffic load [19, 28, 31, 34]. The major inconvenient of this kind of strategies is still the lake of robustness against traffic variations.

In this paper, we propose a new model for fixed channel assignment (FCA) which takes into account both spatial and temporal variation of traffic in order to improve network capacity and frequency plan robustness. In this model, we assume that we have data on traffic variation for $n p$ future periods representing the cyclical evolution of traffic (daily or weekly). The objective is then to find an assignment of the available frequencies to the base stations in order to satisfy traffic demands and to minimize interference. Three criteria are retained to measure the performance of frequency plans: (1) total interference over the given time period, (2) temporal robustness of the frequency plan, and (3) spatial repartition of interference. The first criterion is well known; the other two criteria, however, are rarely studied in the literature. Contrary to reactive and proactive schemes, this model has the advantage of introducing neither additional signaling loads nor interventions on network equipment.

The classical FCA is known to be a very difficult optimization problem $[1,6,13$, $17,27,30]$. The presence of additional optimization criteria in the new model makes the problem even harder. For the resolution purpose, we have elaborated an original genetic Tabu search heuristic (GTS). The main characteristic of the algorithm is the use of a common Tabu list for all population individuals. This structure allows experience sharing between current solutions. Another important feature is its domainspecific genetic operators: violation-directed mutation and geographical crossover [34]. The efficiency of the hybrid algorithm is compared to three other algorithms, derived by switching off some of its components. The first one (noted GLS) is the same genetic Tabu search except that Tabu search is replaced by a simple descent algorithm based on the same neighborhood structure. The second algorithm used for the comparison is a basic Tabu search (TS) algorithm that uses again the same neighborhood structure as before and proceeds by inspecting the entire neighborhood at each iteration. The third algorithm is a hybrid genetic Tabu search algorithm iden- 
tical to GTS algorithm but where the population individuals don't share a common Tabu list. Consequently, each individual have its proper Tabu list.

The paper is organized as follows. In the next section, we describe the context of this study and we give an overview on fixed channel assignment modeling history. Then the impact of traffic variation on frequency plan performance is shown. In the Sect. 3, we introduce formally the multi-criteria cost model. Description of the genetic Tabu search and its operators are presented in Sect. 4. Sections 5, 6 and 7 present the three comparison algorithms. Section 8 is dedicated to experiments and computational results. The conclusion is drawn in the last section.

\section{Fixed channel assignment in GSM networks}

In radio mobile networks [24, 29], communications are ensured by a radio link. The mobile network operators dispose of a very limited number of frequencies to cover all the network area (limited to 62 frequencies in France). For this reason, frequency reuse is indispensable to increase the capacity of a network.

A GSM air-interface [23] is composed of a set of sites, each supporting one to three stations. Each station covers an area called cell representing all the points served by this station (Fig. 1). Fixed channel assignment (FCA) is widely used in today's cellular networks because of its implementation simplicity. In this scheme, each station requires a fixed number of frequencies according to its traffic load, which may simultaneously occur in the cell. Channel assignment consists in finding a permanent assignment of the available frequency spectrum to the stations of the network, which satisfies stations demands and minimizes interference. Interference is caused by the presence of overlapping areas between cells where several signals of good quality are received. ${ }^{1}$ In these areas, traffic satisfaction is highly conditioned by used fre-

Fig. 1 Cellular network map: each colored area corresponds to one cell

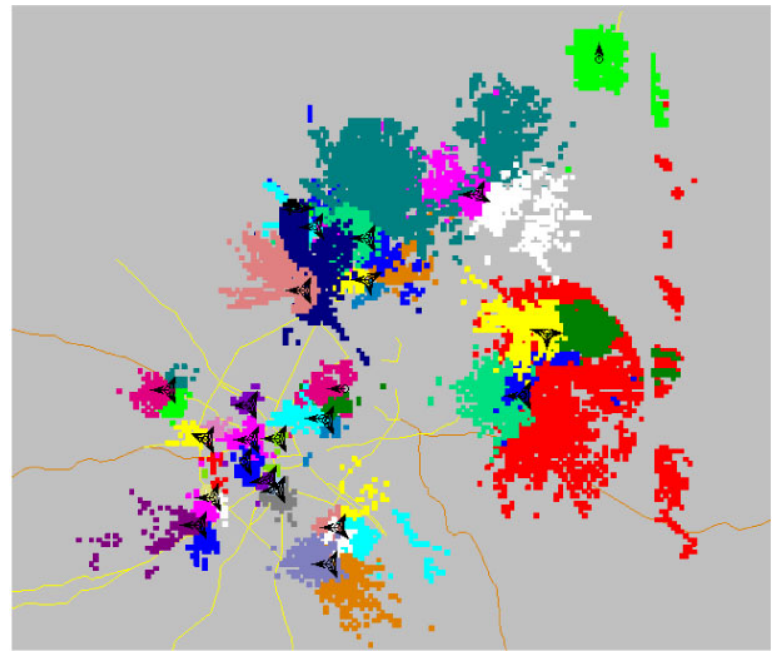

\footnotetext{
${ }^{1}$ Notice that signal overlapping is necessary to ensure communications handovers.
} 
quencies. We distinguish three classes of interference constraints according to their importance:

- Co-station constraint (call it $\mathrm{C} 1$ hereafter): frequencies assigned to the same station must be spaced by at least 3 channels.

- Co-site constraint (call it C2 hereafter): frequencies assigned to stations located on the same site must be spaced by at least 2 channels.

- Inter-site constraint: frequencies assigned to stations belonging to different sites are spaced according to their mutual interference.

Generally, the co-station and co-site interferences are considered as hard constraints that satisfaction conditions the feasibility of the frequency plan, whereas the inter-site interferences are seen as soft constraints that express a preference of some solutions.

The first works on the FCA are based on a reusing matrix [12, 16, 18, 21] indicating channel separation required between frequencies to completely eliminate the interference. In such a model, interference priorities are ignored. In fact, inter-site interference involves different levels of damage to the affected communications. This damage is measured according to used frequencies distance, interference power and the affected traffic. More realistic models were recently proposed, which are based on the quantification of interference risks $[6,9,20]$. In this case, the interference damage produced by each station on each other is estimated. Frequency plan quality corresponds then to the sum of recorded interference damage rather than the number of unsatisfied separation constraints.

The impact of traffic on interference is twofold. As jamming station, traffic intensity describes the using rate of frequencies assigned to the station and hence impacts on the quantity of generated interference. As interfered station, traffic intensity reflects the importance of the area covered by the station and consequently the interest of interference reduction on this area. Since traffic is variable, frequency plan considered good according to traffic situation at a given period might become bad at other periods. To manage traffic evolution, current frequency planning methods are based on an over-sizing of traffic data. One commonly used technique is based on traffic data at second busy hour of the day $(2 \mathrm{BH})$ [21]. More precisely, on each station, the quantity of traffic recorded at the second busy hour of the day is retained (Fig. 2). This traffic is considered constant and occurring in a simultaneous way. This over-sizing involves an inaccuracy in modeling of traffic capacity.

Contrary to aggregating traffic data, we propose here a frequency channel assignment model, which takes into account the dynamic aspect of traffic and is, hence, more adequate in modeling global quality of the frequency plans and their robustness towards these changes. In the following of the article we refer to this model by dynamic model.

In the dynamic model, we consider that traffic evolution follows a cyclical scheme (daily or weekly) that remains stable for a relatively long period (about 3 to 6 months). Consequently, the frequency plan is built to fit the traffic evolution. Such a frequency plan will remain operational for several months. As for classical model, the frequency plan will be readjusted when important changes occur in the cyclical variation of traffic (for example seasonal changes). 


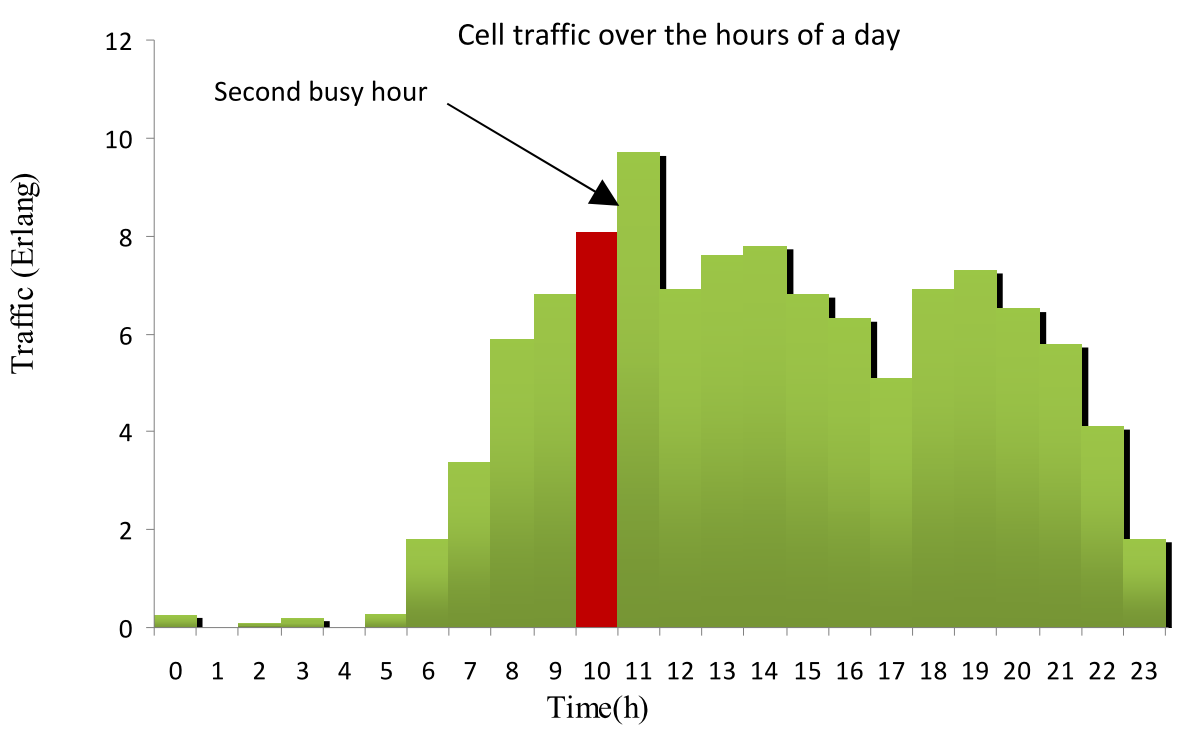

Fig. 2 Traffic evolution of one cell over 24 hours

\section{Fixed channel assignment model}

Let $N$ be a GSM network composed of $n$ stations and $\{1, \ldots, n f\}$ the set of available frequencies. For each station $i$, we know the required number of frequencies, $M A_{i}$, and the traffic load $t_{i}^{h}$ at any period $h \in[1 . . n p]$. We note $t_{i}^{2 B H}$ the traffic load on the station $i$ at the second busy period and $f_{i, k}$ the $k$ th frequency assigned to station $i$.

Let $C 1$ and $C 2$ two binary functions representing the co-station and co-site constraints and are defined as follows:

$$
\begin{gathered}
C 1(i, k, p)=\left\{\begin{array}{ll}
1 & \text { if }\left|f_{i, k}-f_{i, p}\right|<3 \\
0 & \text { else }
\end{array},\right. \\
C 2(i, j, k, p)= \begin{cases}1 & \text { if }\left|f_{i, k}-f_{j, p}\right|<2 \\
0 & \text { else }\end{cases}
\end{gathered}
$$

Let $I$ be the function measuring inter-site interference damage generated by one station on one other. $I\left(i, j, t_{i}, t_{j}, d\right)$ designates interference damage produced by the station $i$ on the station $j$ when they use two frequencies spaced by $d$ channels. Where $t_{i}, t_{j}$ correspond to traffic load on the two stations. The value of the function doesn't correspond directly to a physical measure but it is well correlated with the notion of lost traffic.

In our model, the problem is formulated by $n p$ directed graphs, one per period. Each graph $G^{h}$ represents inter-site constraint damages measured according to traffic situation at period $h$. Nodes of graph correspond to stations and arcs represent interference risks. Each arc linking $i$ to $j$ is weighted by a pair of values $\left(B_{i, j, 0}^{h}, B_{i, j, 1}^{h}\right)$, where:

$$
B_{i, j, d}^{h}=I\left(i, j, t_{i}^{h}, t_{j}^{h}, d\right)
$$


Let us first notice that inter-site interference damage is considered negligible when $d$ exceeds 1 channel. Secondly, co-site and co-station constraints are constant during all the periods since they refer to network architecture and not to clients demand.

On the basis of those graphs, the quality of a frequency plan is assessed on both a global and local level. The global quality of the frequency plan refers to the total interference recorded on the network over the whole time period. The local quality measures the performance stability of the frequency plan over the time and the space. Three criteria are to be retained then: Total interference, frequency plan robustness, and spatial repartition of interference. These criteria can be stated more formally as follows.

- Total interference or global quality of the frequency plan.

$$
F_{1}=\sum_{h=1}^{n p} \sum_{\substack{i=1, j=1 \\ i \neq j}}^{n, n} \sum_{\substack{\left(f_{i, k}, f_{j, p}\right) \\ k \in\left[1 . . M A_{i}\right] \\ p \in\left[1 . . M A_{j}\right]}} B_{i, j,\left|f_{i, k}-f_{j, p}\right|}^{h}
$$

- Robustness of the frequency plan through time periods. It aims to minimize the worst performance of the frequency plan over the time.

$$
F_{2}=\underset{h=1}{M A} \sum_{\substack{i=1, j=1 \\ i \neq j}}^{n, n} \sum_{\substack{\left.f_{i, k}, f_{j, p}\right) \\ k \in\left[1 . . M A_{i}\right] \\ p \in\left[1 . . M A_{j}\right]}} B_{i, j,\left|f_{i, k}-f_{j, p}\right|}^{h}
$$

- Spatial repartition of interference through the network. It aims to minimize the maximal amount of interference on one station.

$$
F_{3}=\underset{i=1}{M A X} \sum_{\substack{j=1 \\ j \neq n}}^{n} \sum_{h=1}^{n p} \sum_{\substack{\left.f_{i, k}, f_{j, p}\right) \\ k \in\left[1 . . M A_{i}\right] \\ p \in\left[1 . . M A_{j}\right]}} B_{i, j,\left|f_{i, k}-f_{j, p}\right|}^{h}
$$

According to this model, the objective of the channel assignment problem is to find the $f_{i, k}$ values which satisfy co-station and co-site constraints and minimize $F_{1}, F_{2}$ and $F_{3}$. The frequency plan will be then operational during all periods. The general scheme of problem modeling and solving is described in Fig. 3.

The computational complexity of the standard FCA problem has been studied in several works $[1,6,8]$. From these studies we know that:

1. FCA, reduced to the minimization of the function $F_{1}$, is NP-complete.

2. The general problem of finding a feasible solution satisfying co-station and co-site constraints is NP-complete.

3. The problem of deciding whether an instance of the problem presents a feasible solution is NP-complete.

In addition, the non-linear nature of the $F_{2}$ and $F_{3}$ criteria increases the difficulty of the problem. Moreover, previous studies have also shown the difficulty of 


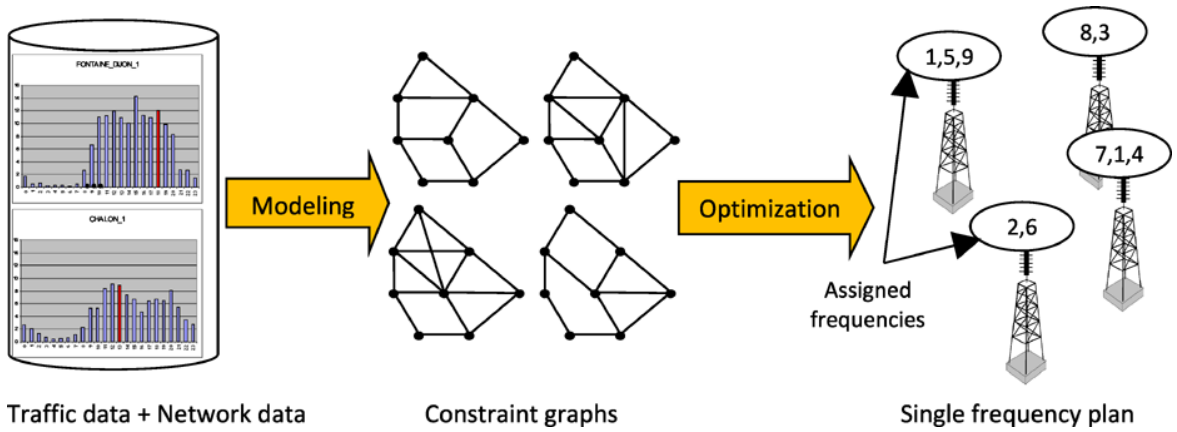

Fig. 3 Fixed channel assignment model: from traffic data evolution over $n p$ periods, $n p$ constraints graphs are built. Constraints graphs are used to construct the fixed frequency plan

the practical solving of the FCA. For these reasons, heuristics constitute a natural and practical solution approach for tackling FCA with the dynamic model.

Next section presents our hybrid heuristic algorithm for solving the FCA problem. This algorithm combines genetic search and a Tabu algorithm and uses the above quality functions $\left(F_{1}, F_{2}\right.$ and $\left.F_{3}\right)$ as part of its evaluation function.

\section{Genetic Tabu search for FCA (GTS)}

Our solution method is based on the hybrid evolutionary framework which combines local search with population based evolutionary algorithms. This framework, which becomes more and more popular in recent years, has been applied with great success to several well-known NP-hard combinatorial problems [11, 24, 26, 32]. Such a hybrid evolutionary algorithm often requires two complementary key elements: a powerful local search operator and a problem specific crossover operator. The crossover operator is used to create new and potentially interesting solutions, which are further improved by the local search operator.

For the channel assignment problem, we developed such a hybrid algorithm, which embeds a Tabu search (TS) procedure [14] within a genetic algorithm [15]. In what follows, we give a detailed presentation of this genetic Tabu search (GTS) algorithm.

\subsection{Individual representation and fitness evaluation}

A frequency plan is coded by a vector $\left\langle f_{1,1}, \ldots, f_{1, M A_{1}}, f_{2,1}, \ldots, f_{2, M A_{2}}, \ldots\right.$, $\left.f_{N, 1}, \ldots, f_{N, M A_{N}}\right\rangle$, representing frequencies assigned to each station. Each element $f_{i, k}$ designates the $k$ th frequency assigned to the station $S_{i}$. The search space of a problem corresponds therefore to all such configurations where $f_{i, k} \in[1 . . n f]$.

Co-station and co-site constraints are handled using a penalty-based approach. The evaluation (fitness) function corresponds to a linear sum of $C_{1}, C_{2}$ and the three criteria $F_{1}, F_{2}$ and $F_{3}(7)$. To emphasize their importance, co-station and co-site constraints are weighted by a large value $\omega$. This value is chosen in such a way that constraints satisfaction has always priority over optimization criteria. To that end, the value of $\omega$ is set to the greatest evaluation of the function $F_{1}+F_{2}+F_{3}$ in the initial population. Since this value is necessarily very high during the initial generation, 
Fig. 4 Working scheme of the Genetic Tabu search algorithm

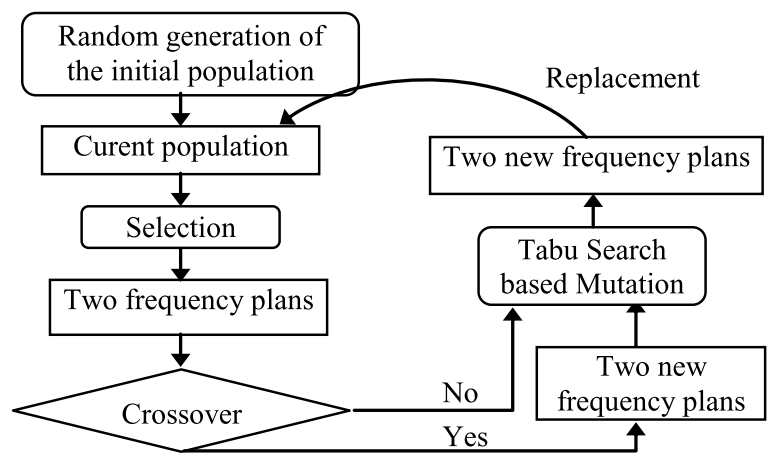

this ensures us that the value of $\omega$ would be large enough to favour hard constraints satisfaction. Notice that the $\omega$ value is kept fix during the search.

$$
\begin{aligned}
F= & \omega\left(\sum_{i=1}^{N} \sum_{k=1}^{M A_{i}-1} \sum_{p=k+1}^{M A_{i}} C 1(i, k, p)+\sum_{i=1}^{N-1} \sum_{\substack{j=i+1 \\
i, j \in \text { same site }}}^{N} \sum_{k=1}^{M A_{i}} \sum_{p=1}^{M A_{j}} C 2(i, j, k, p)\right) \\
& +F_{1}+F_{2}+F_{3}
\end{aligned}
$$

The objective of the FCA algorithm is thus to find the $f_{i, k}$ values that minimize the function $F$. Notice that F1, F2 and F3 have a unit weight. This is for the following reasons.

- The three functions are of identical nature. All correspond to an interference quantity.

- The normalization of the functions will be prejudicial to F1 criterion since it corresponds to the biggest values. However the analysis of the three criteria shows that the decision maker is principally interested in improving the global quality of the network.

\subsection{General scheme of the GTS algorithm}

Starting from an initial population of random frequency plans (the population size is fixed to 10 for all the referenced tests), the genetic Tabu search algorithm performs a series of cycles called generations. At each generation, two frequency plans are selected from the current population and combined with a problem specific crossover operator (section D). Then the two generated frequency plans are mutated by a Tabu search operator (section E) before being inserted into the population. Figure 4 presents schematically the working of the algorithm.

\subsection{Selection and replacement operators}

To favor the selection of good solutions, population individuals are ordered according to their fitness so that the best solution (lower fitness) has the rank 0 , the second best solution obtains the rank 1 and so on. The worst solution will thus obtain the 
biggest rank (population size -1 ). Let $r_{i}$ be the rank of the individual $i$, the selection probability of $i$ is then calculated following (8).

$$
S P_{i}=\frac{\left(P o p \_s i z e-r_{i}\right) \times 2}{\text { Pop_size } \times(\text { Pop_size }+1)}
$$

Rank based selection has a major advantage to regulate selection pressure and hence allows better convergence of the algorithm [36]. By convergence we mean the stagnation state of the search process caused by the resemblance between population individuals. Using the rank based selection, we ensure that the selection probability of the best solution doesn't exceed the value $2 /\left(P o p \_s i z e+1\right)$. Therefore reproduction rate of parents in further generations is limited and convergence process is better controlled. In addition, ranking eliminates the need for fitness scaling when the individual's fitness within the population converges to a narrow interval and hence selection pressure is maintained during the search. This approach shares similarities with the fitness entropy based method used in [35].

New individuals, created by crossover and then mutation (next 2 sections), are inserted in the population in place of other solutions (not necessarily their parents). Replacement favors the elimination of bad frequency plans. Equation (9) gives the replacement probability of an individual $i$.

$$
R P_{i}=\frac{r_{i} \times 2}{P o p \_s i z e \times\left(P o p \_s i z e-1\right)}
$$

Let us notice that the best frequency plan is never replaced. This elitism selection strategy ensures that the best solution found during the search will not be lost and will continue to contribute to further evolutions.

\subsection{Crossover operator}

Several crossover operators are available in the literature. However, in several cases the use of domain-specific operators has given better results. The analysis of the frequency plan structure shows the importance of the notion of vicinity. In fact, the quality of a frequency plan depends on the frequencies distribution at the local level (cell, site, district, etc. ... ). A good crossover operator should allow the conservation of the conflict-free assignments of the parent solutions and help the resolution of conflicting assignments.

For that reason, we have adopted the so-called geographic crossover described in [34], which is specially designed for channel assignment. This crossover operator works as follow. Given two frequency plans, the first step of the crossover consists in taking randomly a reference station $S_{i}$. Let $V\left(S_{i}\right)$ be the set of co-site and interfering stations of $S_{i}\left(S_{j}\right.$ interferes with $S_{i}$ if $\exists d$ and $h / B_{i, j, d}^{h} \neq 0$ or $B_{j, i, d}^{h} \neq 0$ where $B_{i, j, d}^{h}$ definition is given in (3). Then the frequencies assigned to stations $S_{i} \cup V\left(S_{i}\right)$ are exchanged between the two parents generating two new frequency plans (Fig. 5). In that manner, this geographic crossover helps conserve the building blocks constituting parent codes. This is made by swapping information related to the local resolution of interference between stations. 


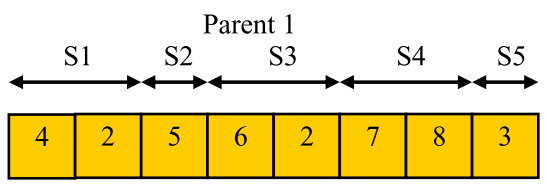

Parent 2

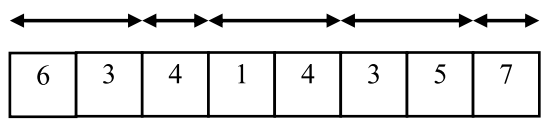

Offspring 1
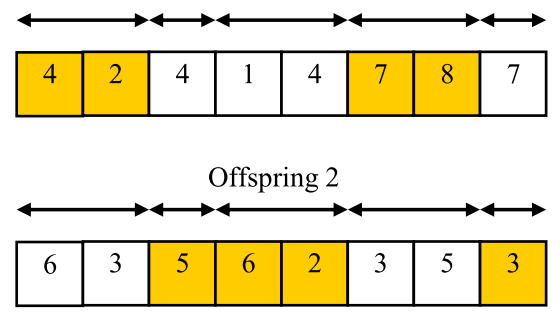

Fig. 5 Example of crossover between two frequency plans. Values of a vector present the frequencies allocated to each station. Frequencies assigned to the same station are indicated by an horizontal double arrow. The interference risks are: S1 interferes with S2, S1 interferes with S4, S2 interferes with S3, S3 interferes with S5 and S4 interferes with S5. In the example we consider that the reference station is S3 and since it interferes with $\mathrm{S} 2$ and $\mathrm{S} 5$, the frequencies assigned to stations $\{S 2, S 3, S 5\}$ are exchanged

We give here the skeleton of crossover operator.

Crossover (Frequency plan parent1, parent2)

Begin

St_ref $=$ random(nbstations)

Neighbors_St $=$ St_ref + co_site_station $\left(S t \_r e f\right)+$ interfer(St_ref);

for each $i$ belong to Neighbors_St

for each $k$ an assignment of $i$ offspring $2[i, k]:=$ parent $1[i, k]$; offspring $1[i, k]:=$ parent $2[i, k]$;

End for End for

for each i not belong to Neighbors_St

for each $k$ an assignment of $i$ offspring $1[i, k]:=$ parent $1[i, k]$;

\section{End for}

\section{End for}

$$
\text { offspring } 2[i, k]:=\text { parent } 2[i, k] \text {; }
$$

\section{End.}

$$
\text { Return (offspring1, offspring2) }
$$

\subsection{Tabu search based mutation}

For mutation, we use a local search operator based on Tabu search (TS). The purpose of the TS operator is to improve iteratively the frequency plans generated by the crossover before inserting them into the population. The TS operator shares some similarities with the Tabu algorithm described in [17] which is designed for solving the FAP based on the static traffic model. However, our TS operator distinguishes itself by some specific features: the way of assessing the fitness of frequency assignments, the manner that the moves are chosen and the role of the Tabu list.

Tabu search based operator corresponds to a kind of macro-mutation where at each iteration (also called a move), a conflicting assignment $(i, k)$ representing the $k$ th 
Fig. 6 Common Tabu list mechanism

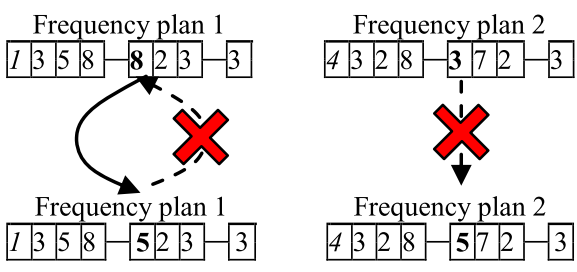

frequency assigned to station $S_{i}$, is chosen and its current frequency value is replaced by another value. More precisely, these choices are taken following two steps.

1. The first step consists in choosing the assignment to change. For that end, a violation score is first calculated for each assignment $(i, k)$. This score measures the contribution of this assignment to the recorded interference. The violation scores are used as a way to locate the problematic affectations. Equation (10) gives the expression of the violation score of the $k$ th frequency assigned to station $S_{i}$. Once the violation scores are calculated, we choose an assignment according to (11), which describes the choice probability of an assignment $(i, k)$. Therefore assignments with a high violation score will have more chance to be mutated.

$$
\begin{aligned}
\operatorname{SCORE}_{i, k}=\omega \times\left(\sum_{\substack{p=1 \\
p \neq k}}^{M A_{j}} C 1(i, k, p)+\sum_{\substack{j=1 \\
j \neq i \\
j \neq \in \text { same site }}}^{N} \sum_{p=1}^{M A_{j}} C 2(i, j, k, p)\right) \\
+\sum_{h=1}^{n p} \sum_{\substack{j=1 \\
j \neq i}}^{N} \sum_{\substack{f_{j p} \\
p \in\left[1 . . M A_{j}\right]}} B_{i, j,\left|f_{i k}-f_{j p}\right|}^{h} \\
\operatorname{GSP}_{i, k}=\operatorname{SCORE}_{i, k} / \sum_{j=1}^{N} \sum_{p=1}^{M A_{j}} \operatorname{SCORE}_{j, p}
\end{aligned}
$$

2. After the choice of an assignment $(i, k)$, a new frequency value $f_{i, k}^{\prime}$ is affected to it to replace the current value $f_{i, k}$. The chosen $f_{i, k}^{\prime}$ corresponds to the frequency value leading to the best frequency plan and not being forbidden by the Tabu list. The elements $\left(i, k, f_{i, k}\right)$ and $\left(i, k, f_{i, k}^{\prime}\right)$ are then added to the Tabu list.

This process is repeated for a given number of iterations noted TSML.

The Tabu list is implemented as an attributive memory, where certain attributes of the frequency plan are stored instead of the full solution. The Tabu list is then handled as a FIFO list. The number of iterations that a move is considered Tabu (Tabu tenure) is static and corresponds to the Tabu list size.

The new Tabu elements have two different roles. The element $\left(i, k, f_{i, k}\right)$ prevents the algorithm from re-visiting previously seen solutions. The element $\left(i, k, f_{i, k}^{\prime}\right)$ prevents the other individuals from re-exploring the same search area since the Tabu list is shared by all the individuals as shown in Fig. 6. 
The number of TS iterations is determined by the TSML (Tabu search based Mutation Length) parameter. The result of the Tabu search based mutation is the best frequency plan encountered during the Tabu search cycle.

We give below the main loop of the Tabu search based mutation followed by the genetic Tabu search algorithm. The main procedure is stopped when the genetic Tabu search reaches a given number of iterations. During the experimental tests, this number is chosen in such a manner that it guaranties the same running time for all tested algorithms. Notice that a criterion like "stagnation state condition" is not able to give such a guarantee.

TabuSearchOperator(Frequency plan fp)

Begin

Best_fp :=fp;

CalculateScores(fp);

for iter := 1 to TSML $\{$ Tabu Search based mutation length $\}$

$(i, k):=$ ChooseAssignment(fp);

f_old $:=\mathrm{fp}[i, k]$;

$\mathrm{f} \_$new $:=$ChooseFrequency $(\mathrm{fp}, i, k)$;

AddToTabuList $(i, k$, f_old);

AddToTabuList $\left(i, k, \mathrm{f} \_\right.$new $)$;

$\mathrm{fp}[i, k]:=\mathrm{f} \_$new;

UpdateScores(fp);

If BetterThan(fp,Best_fp)

then Best_fp = fp; End if

End for

End.

Genetic Tabu Search

Begin

$\mathrm{P}:=$ RandomInitPopulation(Pop_size);

For $\mathrm{g}:=1$ to $\mathrm{NbGenerations}$

$(\mathrm{p} 1, \mathrm{p} 2):=$ SelectParents(P)

with a Pc probability do

$(\mathrm{f} 1, \mathrm{f} 2):=$ Crossover $(\mathrm{p} 1, \mathrm{p} 2)$

otherwise $\mathrm{f} 1:=\mathrm{p} 1 ; \mathrm{f} 2:=\mathrm{p} 2$;

$\mathrm{f} 1:=$ TabuSearchOperator(f1);

$\mathrm{f} 2:=$ TabuSearchOperator(f2);

$(\mathrm{v} 1, \mathrm{v} 2):=\operatorname{SelectVictims}(\mathrm{P})$;

ReplaceBy(v1,f1);

End for

ReplaceBy(v2,f2);

End.

4.6 Synergy between Tabu search based mutation and crossover

As the generations go by and thanks to selection and crossover mechanisms, the population individuals tend to present the same properties. Considering the directive aspect of the Tabu search based mutation, similar individuals are induced to follow the 
same itinerary. Consequently, this involves an exploration redundancy and therefore the slowness and the loss in diversity of search. To overcome this problem, Tabu list stores the recent evolution trace of all population individuals. The itinerary followed by an individual is avoided for the others involving the multiplicity of optimization itineraries. The crossover operator appears then as the only mechanism to share and exchange experience between individuals. In other words, the crossover operator allows the combination of various search itineraries.

Crossover operator can be considered as an exploitation tool in our hybrid algorithm. It leads to individuals that bring no diversity into the population since all the genes are inherited from one of parents but at the same time offspring individual cannot be considered in the local neighbor of their parents since they are so different of each parent taken separately. The Tabu search based mutation operator, as for it, proceeds locally by operating small modifications of the solutions. The use of the common Tabu list mechanism allows not only to avoid the exploration of already visited area by the same individual but also all areas visited by the other individuals. Therefore, despite that the Tabu search based mutation is a local search procedure it remains a good way for exploration since it has a global view of the search state given by the Tabu list. Consequently, there is no redundancy in the roles of crossover and mutation operators. The mutation works as a life cycle during which each individual acquire an experience that enriches the group experience. This is possible thanks to the common Tabu list that prevents each individual from following the same search itinerary. The crossover operator appears here as the unique way to exchange this experience between individuals.

\section{Tabu search for FCA (TS)}

In order to assess the hybridizing effect of our genetic Tabu search algorithm, we have also developed a separate Tabu search algorithm [14, 37]. This algorithm is basically the same as the Tabu search based mutation (Sect. 4.5) since the most important components are shared (individual representation, evaluation function, Tabu list management etc.) Tabu search is a meta-heuristic well known for its ability to escape from local optima. The last decade has seen the multiplication of works on Tabu search in the field of telecommunications and notably radio networks [25, 39]. Tabu search is a local heuristic based on the four principals:

- Define the manner to construct the neighborhood of a solution and the kind of move to use.

- An interdiction rule of a set of moves (considered Tabu) generally to prevent search cycles.

- A rule of choosing one solution among the set of neighbors.

- An aspiration criterion allowing the use of a move even if it is Tabu.

\subsection{Initial solution}

The Tabu search algorithm starts from an initial solution generated using an adaptation of the DSATUR procedure [2]. The algorithm assigns frequencies to stations 
progressively. At each step, we choose a free assignment $(i, k)$ having a minimal number of allowed frequencies (frequencies satisfying all interference constraints: co-station, co-site and inter-site). Among all the allowed frequencies, we choose the one $f_{i, k}$ that has the minimal index. If there is no allowed frequency, we choose then one frequency that satisfies co-station and co-site constraints and minimizes inter-site interferences.

\subsection{Neighborhood}

For a given frequency plan, the set of neighbors corresponds to all possible frequency plans which can be obtained by changing the value $f_{i, k}$ of the $k$ th frequency of a station $i$. Consequently, each move is represented by a triplet $(i, k, f)$ corresponding respectively to the station, the assignment and the new frequency value. At each iteration of the Tabu search algorithm, the neighborhood of the current solution is examined. Since the number of neighbors may be very high $(W \times(n f-1)$ where $\left.W=\sum_{i=1}^{N} M A_{i}\right)$ we adopt an incremental evaluation technique [10]. In this technique, a $n f \times W$ matrix is incrementally calculated. The elements of the matrix represent the cost variation (called also move value) for each possible move of the current solution. At each step, the non-Tabu neighboring solution with the best cost variation is selected and becomes the current solution. If a neighbor is better than the best solution found so far, this neighbor is still selected even if it is forbidden by the Tabu list. After a move, the matrix is updated according to this change and the selected move is inserted in the Tabu list for a given number of iterations.

We give here the skeleton of the Tabu search algorithm.

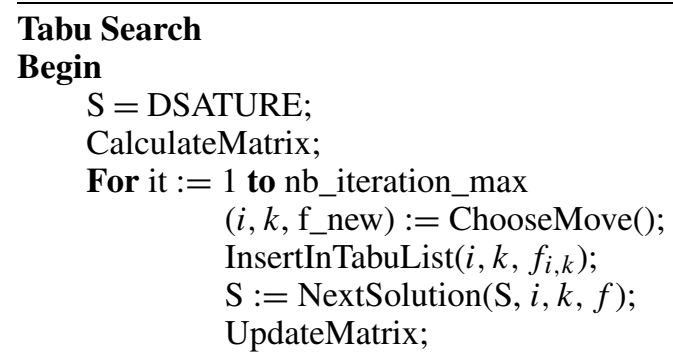

End.

\section{Genetic local search for FCA (GLS)}

In order to assess the effect of the Tabu search based mutation in the hybrid genetic algorithm, in particular to see how the common Tabu list impacts on the search; we have discarded the common Tabu list, the other components remaining the same. In this case, the initial Tabu search based mutation degenerates to a simple descent based mutation. As for the initial genetic Tabu search algorithm, this mutation operator carries out iterative changes on a single frequency plan. At each iteration, the choice of the assignment to change is made on the basis of violation scores. However, changes are no more recorded in the Tabu list. We give here the algorithmic scheme of the descent mutation operator, the main algorithm being the same. 


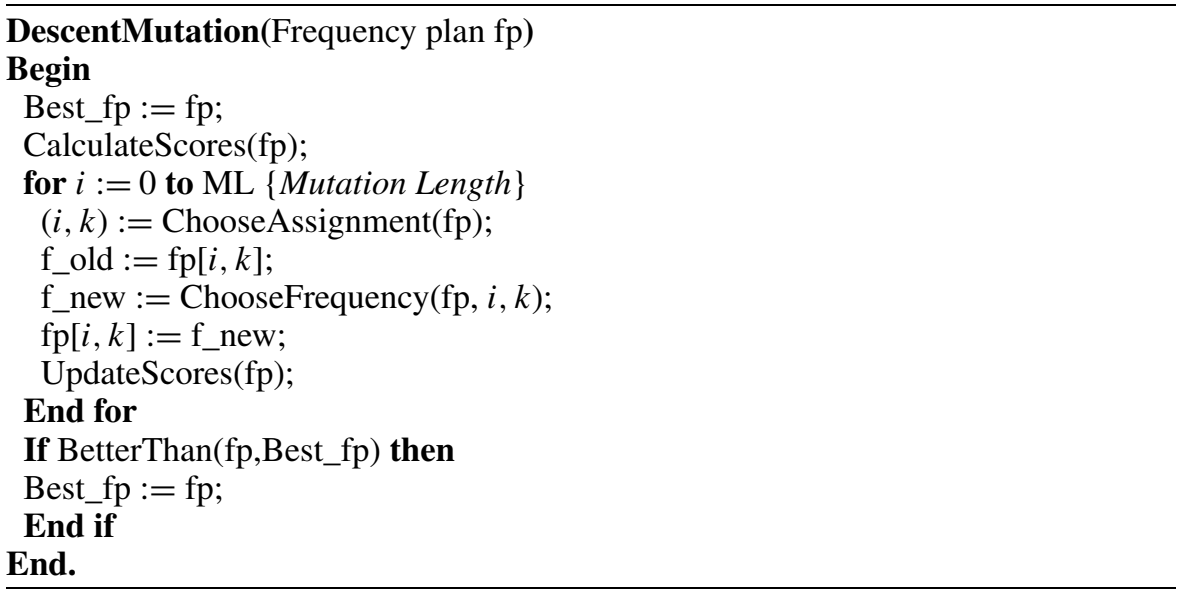

\section{Genetic Tabu search with no common list for FCA (GTS_NCL)}

To support the comparison we also compare our GTS algorithm with an identical algorithm noted GTS_NCL. The common Tabu list is replaced by an individualized short-term Tabu list. More precisely, at the beginning of each Tabu search based mutation; the Tabu list is set to empty. Therefore each mutation execution is completely independent of the rest of the search.

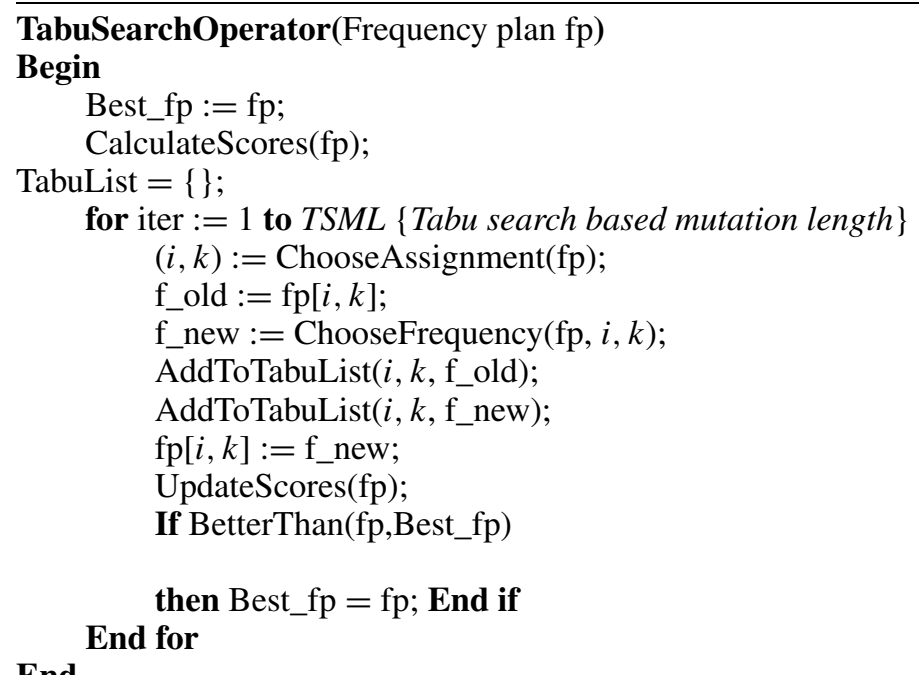

\section{Experimental results}

Experimental tests presented in this section have two purposes. At the algorithmic level, we assess the performance of the Genetic Tabu algorithm and the influence 
of algorithmic parameters on the search. In particular, we study the impact of Tabubased mutation on the hybrid algorithm. At the modeling level, we compare frequency plans generated using our traffic model with those generated using the classical $2 B H$ modeling [21].

Tests are carried out on both fictitious and real world problems and are accessible at http://www.info.univ-angers.fr/pub/hao/COAP2010.html. The two fictitious FCA instances used in our experimentation represent 63 stations extracted from a real network $B$. The word "fictitious" refers only to the data of traffic evolution. The two instances have the following characteristics: 225 frequencies to assign, traffic data over 6 periods and around 1100 inter-site constraints. Each instance represents a different class of traffic evolution that allows us to study the performance of dynamic traffic modeling on different traffic evolution scenarios.

The first network, $B \_63 \_1$, represents synchronous and proportional rises and falls of traffic on the entire network. The second instance, $B \_63 \_2$, emphasizes the mobility aspect of clients and presents two distinct areas. The rise of traffic on one area is accompanied by a fall of traffic intensity on the other area.

The third network (Network $D$ ) uses real traffic evolution data. This network is characterized by 639 stations, 1411 frequencies to assign, about 30000 inter-site constraints and traffic data over 13 hours (7:00-20:00). The studied period corresponds to the overloaded hours of the network, the other non-problematic hours being neglected.

In addition to these three instances, two other instances are used which are constructed by logarithmic scaling of the weights of $B \_63 \_1$ and $B \_63 \_2$ graphs $\left(B_{i, j, d}^{\prime} h=\log _{10}\left(B_{i, j, d}^{h} \times 100+1\right)\right)$. We note by $B \_63 \_1 \_l o g$ and $B \_63 \_1 \_l o g$ these problem instances. Logarithmic scaling allows homogenizing of interference weights and therefore the study of the performance of algorithms under homogeneous edges weights.

Finally we have implemented a random graph generator (RGG). This tool allows us to generate a graph by specifying the number of stations, the number of periods as well as the density and the heterogeneity degree of the graph. The density of the graph is taken from $[0,1]$ measuring the probability that two different stations are interfering. The heterogeneity parameter is a value between $[0,1]$ defining the heterogeneity level of the graph weights. Four problem instances are generated using the RGG; all these instances are composed of 50 stations with 5 periods. The first instance (RGG_50_1) presents a density of 0.5 and a heterogeneity level of 0.2 . The second one, RGG_50_2 has a density of 0.2 and a heterogeneity level of 0.5 . RGG_50_3 has a density of 0.2 and a heterogeneity level of 0.2 . The fourth instance has a density of 0.2 and a heterogeneity level of 1.0 .

For all these networks, we consider that the same scheme of traffic evolution will recur in the future days. The frequency plan that fits the traffic evolution over the studied period will fit the traffic of the network in the future days until an important traffic evolution occurs. At that moment, the frequency plan will be changed. 
Table 1 Average and standard deviation on 30 runs of the solutions costs found by the GTS in function of TLS. The hard constraints are always satisfied by all the runs

\begin{tabular}{rlllllll}
\hline \multirow{2}{*}{$T L S$} & B_63_1 & & & & B_63_2 & \\
& Mean & SD & SD \% & & Mean & SD & SD \% \\
\hline 20 & 557718 & 25045 & 4.6 & 504325 & 29247 & 5.8 \\
50 & 546582 & 31105 & 5.6 & 498448 & 20491 & 4.1 \\
100 & 532261 & 26826 & 5.0 & 445287 & 28418 & 6.4 \\
$\mathbf{2 0 0}$ & $\mathbf{5 2 3 7 8 6}$ & $\mathbf{2 9 2 9 5}$ & $\mathbf{5 . 5}$ & $\mathbf{4 2 8 8 9 4}$ & $\mathbf{2 2 7 9 6}$ & $\mathbf{5 . 3}$ \\
500 & 549305 & 26150 & 4.8 & & 460561 & 21645 & 4.7 \\
1000 & 550681 & 29728 & 5.4 & 485127 & 25230 & 5.2 \\
2000 & 555640 & 30868 & 5.6 & 508254 & 29714 & 5.8 \\
\hline
\end{tabular}

Table 2 Average and standard deviation on 30 runs of the solutions costs (Function F) found by the GTS in function of $T S M L$. The hard constraints are always satisfied by all the runs

\begin{tabular}{rlllllll}
\hline TSML & B_63_1 & & & & B_63_2 & \\
& Mean & SD & SD \% & & Mean & SD & SD \% \\
\hline 5 & 553125 & 26627 & 4.8 & & 522485 & 15868 & 3.3 \\
10 & 529566 & 16121 & 3.1 & & 491515 & 14548 & 2.9 \\
$\mathbf{3 0}$ & $\mathbf{5 2 3 4 8 6}$ & $\mathbf{1 8 0 5 9}$ & $\mathbf{3 . 4}$ & & $\mathbf{4 2 8 8 9 4}$ & $\mathbf{2 1 1 0 6}$ & $\mathbf{4 . 9}$ \\
100 & 533429 & 17816 & 3.3 & & 436773 & 15872 & 3.6 \\
200 & 540882 & 16981 & 3.1 & & 438906 & 13371 & 3.1 \\
500 & 547281 & 29309 & 5.3 & & 465713 & 17911 & 3.8 \\
\hline
\end{tabular}

\subsection{Genetic tabu search performance}

The quality of the best solution found in the final population is taken as the final result of a search. To eliminate possible implementation effects, we do not consider the solving speed. Thus search may be run for several hours.

\subsubsection{Influence of search parameters}

In this first part of the study, we are interested in studying the influence of two most important specific parameters for our genetic Tabu search algorithm: Tabu list size or Tabu tenure $(T L S)$ and Tabu search based mutation length (TSML). To observe the influence of $T L S$ on the search we vary its values and we fix the other parameters. Table 1 gives for each value of $T L S$ the average (Mean) and the standard deviation (SD) on 30 runs of the solutions costs (function $F$ ) found by the GTS. The other parameters are fixed as follow: population size $=10$, generation number $=100,000$, $T S M L=30, P c=0.3$. Population size and $P c$ values are fixed according to a primary test phase showing that given its intensification and diversification quality, the GTS algorithm performs well under small population size and moderate use of crossover.

The influence of TSML on the search is analyzed in Table 2. In this case, we keep the same values of population size, generation number and crossover probability and we fix the value of $T L S$ to 200 . Let us mention that hard constraints are always satisfied for every tested value and for all the runs.

From Table 1, we observe that moderate values of $T L S$ (lines corresponding to $T L S=100,200$ and 500) give the best results. In fact, a low value of $T L S$ deteriorates 
Fig. 7 Evolution profiles according to different values of TSML for the B_63_1 instance. Each curve represents an average of the evolution tendencies over 10 runs corresponding to the same $T S M L$ value. The $x$-axes (number of generations) and $y$-axes (fitness function $F$ ) are scaled in $\log$

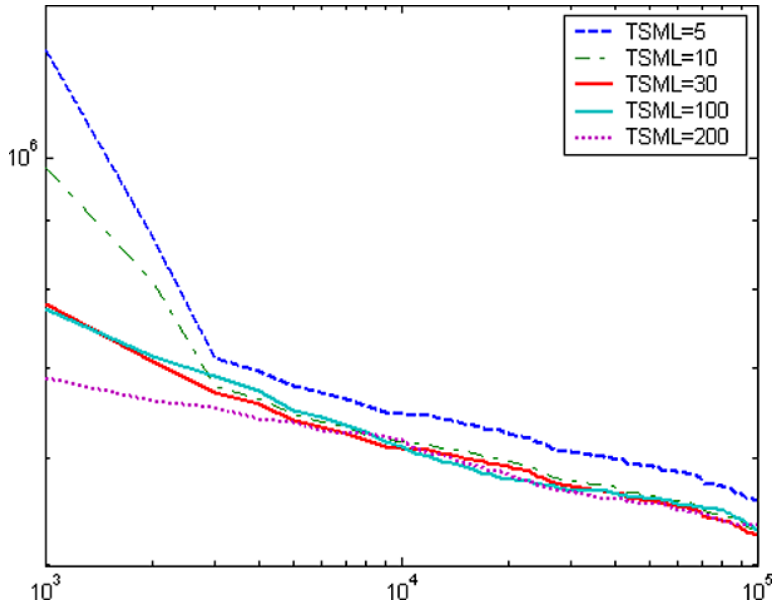

the exploration quality of the search. A high value of TLS slows down the search convergence and restricts its freedom of movement.

In the same way, from Table 2, we observe that the Tabu search length has an important influence on search performances. When Tabu search length is low, Tabu search mutation has no enough time to improve the quality of new solutions generated by crossover. For high values of TSML (against Tabu list size), Tabu search based mutation leads to premature convergence of the search.

Figure 7, presenting the evolution profiles of GTS with different values of TSML parameter, confirms this hypothesis. In fact, the curve corresponding to TSML values 200 shows a fast and premature convergence of search (at generation 1000). The figure shows also that the best solutions are obtained for average values of TSML, which allows a better evolution. Notice that the convergence phenomenon of search is not clearly observable because $\mathrm{x}$-axe and $\mathrm{y}$-axe are scaled in log. Despite of this, one may still observe the difference among the evolution curves at the first generations of the search.

The values of standard deviation given in Tables 1 and 2 indicate the relative stability of the GTS algorithm, since the average deviation of the results doesn't exceed $5.8 \%$ in the worst case.

In Fig. 8, we underline the existing relationship between Tabu search length and Tabu list size. The horizontal axe represents the average quality on 30 runs of obtained solutions. The average is calculated using the same values of the parameters TLS and TSML. Each pair of bars in the histogram corresponds to the values of the two parameters $T L S$ and $T S M L$ chosen for the corresponding experience.

We can observe, for example, that for $T L S=500$, the ideal value of $T S M L$ is 100 , whereas the ideal value is about 200 or 500 when $T L S=2000$. Therefore, the choice of best values of the two analyzed parameters must obey to a certain rule of proportionality. This proportionality must be made in such a manner to allow the sharing of experience between population individuals, experience sharing being ensured by the Tabu list mechanism. 


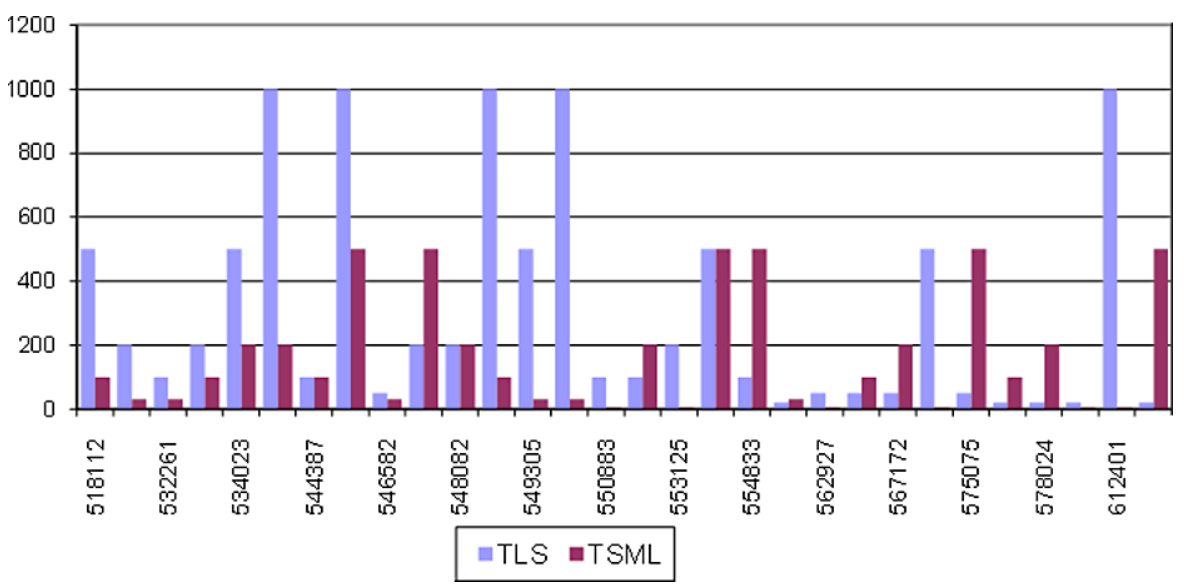

Fig. 8 Quality $F$ in function of Tabu list size and Tabu search length. Abscises correspond to the average cost obtained by each pair of parameters values over 30 runs. Tests are carried out on the B_63_1 instance

\subsubsection{Comparison with TS, GLS and GTS_NCL}

The performance of the Genetic Tabu Search (GTS) algorithm is evaluated on the nine problem instances described before. The results are compared with those obtained by Tabu search algorithm (Sect. 5), Genetic Local Search (Sect. 6) and Genetic Tabu search without common Tabu list (Sect. 7). Table 3 gives for each instance and for each algorithm the quality of obtained results over 30 runs. The column $V C$ corresponds to the average number of violated co-station and co-site constraints. The columns $A V G, M I N$ and $S D$ represent the average, the minimum and the standard deviation of the sum $F_{1}+F_{2}+F_{3}$. On the other side, $T S$ algorithm implements deterministic procedures and therefore only one run is performed on each instance. The performances of the algorithm are represented by the columns $V C$ and $F_{1}+F_{2}+F_{3}$.

The first remark is that the Genetic Local Search algorithm performs the worst. For the nine instances it doesn't satisfy even the hard constraints. This shows clearly the importance of Tabu list mechanism. Tabu search alone achieves better solutions but these results are considerably improved by introducing Tabu search mechanisms into a genetic search.

Therefore, GTS and GTS_NCL prove their efficiency compared with the competing methods. At the same time, we observe that GTS algorithm performs better than GTS_NCL. This shows the interest of the common Tabu list with respect to the individualized Tabu list as in GTS_NCL. The experimental parameters for the nine experiments are: Population size $=10, T S M L=30, T L S=500, P c=0,3$.

Finally, notice that GTS, GLS and GTS_NC have the same time complexity even if GLS requires less memory given that it doesn't require a tabu list.

\subsection{Dynamic modeling performances}

The purpose of this section is to compare our dynamic scheme of fixed channel assignment with the classical scheme based on $2 \mathrm{BH}$ traffic data from an operational 
Table 3 Comparison over 30 runs (10 runs for the instance D) of solutions given by GTS with common Tabu list, GTS with no common Tabu list, GLS and Tabu search for nine problem instances. For each method, we give the average number of violated costation and cosite constraints (VC) as well as the average (AVG), the standard deviation (SD) and the minimum (MIN) of the sum $F=F_{1}+F_{2}+F_{3}$

\begin{tabular}{|c|c|c|c|c|c|c|c|c|c|c|}
\hline & \multicolumn{5}{|c|}{ GTS_CL } & \multicolumn{5}{|c|}{ GTS_NCL } \\
\hline & \multicolumn{3}{|c|}{$\begin{array}{ll}\mathrm{VC} & \text { AVG }\end{array}$} & MIN & SD & \multicolumn{2}{|c|}{$\begin{array}{ll}\mathrm{VC} & \text { AVG }\end{array}$} & \multicolumn{2}{|c|}{ MIN } & SD \\
\hline B_63_1 & 0 & & 8327 & 503832 & 27052 & 0 & 555786 & & 518789 & 24692 \\
\hline B_63_2 & 0 & & 9856 & 423864 & 25522 & 1.4 & 605308 & & 538202 & 66235 \\
\hline $\mathrm{D}$ & 0 & & 3268 & 1339886 & 30862 & 0 & 1402166 & & 1351928 & 42117 \\
\hline B_63_1_log & 0 & & 0022 & 19688 & 215 & 0.3 & 20246 & & 19241 & 1119 \\
\hline B_63_2_log & 0 & & 9590 & 19246 & 270 & 0 & 19447 & & 18678 & 439 \\
\hline RGG_50_1 & 0 & & 2501 & 11903538 & 1273441 & 0 & 12102330 & & 1940577 & 1106978 \\
\hline RGG_50_2 & 0 & & 6256 & 6358412 & 130154 & 0 & 6565898 & & 6373658 & 122767 \\
\hline RGG_50_3 & 0 & & 4705 & 4625048 & 98654 & 0 & 4810656 & & 4650450 & 110548 \\
\hline \multirow[t]{3}{*}{ RGG_50_4 } & 0 & & 4372 & 8452504 & 157898 & 0 & 9161055 & & 9132544 & 121230 \\
\hline & \multicolumn{5}{|c|}{ GLS } & & & \multicolumn{3}{|c|}{ TS } \\
\hline & \multicolumn{2}{|c|}{$\overline{\mathrm{VC}}$} & \multicolumn{2}{|c|}{ AVG } & MIN & \multicolumn{2}{|l|}{ SD } & $\overline{\mathrm{VC}}$ & \multicolumn{2}{|c|}{$F 1+F 2+F 3$} \\
\hline B_63_1 & \multicolumn{2}{|c|}{1.3} & \multicolumn{2}{|c|}{713941} & 610576 & \multicolumn{2}{|c|}{81311} & 1 & \multicolumn{2}{|c|}{518558} \\
\hline B_63_2 & \multicolumn{2}{|c|}{1.5} & \multicolumn{2}{|c|}{781175} & 550439 & \multicolumn{2}{|c|}{291000} & 0 & \multicolumn{2}{|c|}{510530} \\
\hline $\mathrm{D}$ & \multicolumn{2}{|c|}{2.3} & \multicolumn{2}{|c|}{2710251} & 2388847 & \multicolumn{2}{|c|}{61911} & 0 & \multicolumn{2}{|c|}{1624301} \\
\hline B_63_1_log & \multicolumn{2}{|c|}{1} & \multicolumn{2}{|c|}{21648} & 20309 & \multicolumn{2}{|c|}{661} & 0 & & 9720 \\
\hline B_63_2_log & & .1 & & 376 & 20421 & & 701 & 0 & & 8300 \\
\hline RGG_50_1 & 0 & & 1226 & 814 & 12135588 & 118 & 3420 & 0 & 124 & 2360 \\
\hline RGG_50_2 & 0 & & 657 & 387 & 6367725 & & 1153 & 0 & & 0951 \\
\hline RGG_50_3 & 0 & & 496 & 218 & 488056 & & 8845 & 0 & & 3022 \\
\hline RGG_50_4 & 0 & & 936 & 770 & 9220254 & & 4200 & 0 & & 6809 \\
\hline
\end{tabular}

point of view. Comparison between dynamic traffic modeling and $2 \mathrm{BH}$ modeling is made on the basis of lost traffic, measured in Erlang, one Erlang corresponding to one hour of communications. We use quality evaluator of PARCELL ${ }^{\odot 2}$ to measure the lost traffic produced by a given frequency plan. More precisely, given the stations parameters, geographical database, traffic data and a frequency plan, the quality evaluator calculates the lost traffic quantity on each station. Loss in traffic is measured in terms of FER (Frame Erasure Rate). The communication is considered bad if this rate exceeds a given threshold. According to the required radio quality, we distinguish 3 different thresholds: $2 \%, 4 \%$ and $7 \%$.

The classical static model $2 \mathrm{BH}$ uses a single interference graph $G^{2 B H}$ where the weights are calculated on the basis of $2 \mathrm{BH}$ traffic data. The objective function corresponds then to satisfy the imperative constraints and minimizes inter-site interference.

${ }^{2}$ Engineering tool for design of mobile radio network, ORANGE all rights reserved. 
Table 4 Results of classical and dynamic traffic modeling for network $D \_639 \_1$

\begin{tabular}{|c|c|c|c|c|c|c|c|}
\hline \multirow[b]{2}{*}{ Period } & \multirow[b]{2}{*}{ Traffic } & \multicolumn{2}{|c|}{$\begin{array}{l}\text { Lost traffic at } 2 \% \\
\text { FER (Erl) }\end{array}$} & \multicolumn{2}{|c|}{$\begin{array}{l}\text { Lost traffic at } 4 \% \\
\text { FER (Erl) }\end{array}$} & \multicolumn{2}{|c|}{$\begin{array}{l}\text { Lost traffic at } 7 \% \\
\text { FER (Erl) }\end{array}$} \\
\hline & & Classical & Dynamic & Classical & Dynamic & Classical & Dynamic \\
\hline 7:00-8:00 & 504.11 & 5.16 & 4.58 & 2.91 & 2.45 & 1.62 & 1.32 \\
\hline 8:00-9:00 & 1170.02 & 16.74 & 16.06 & 9.39 & 8.57 & 5.06 & 4.47 \\
\hline $9: 00-10: 00$ & 1747.71 & 31.14 & 31.04 & 17.47 & 16.65 & 9.37 & 8.93 \\
\hline $10: 00-11: 00$ & 2017.26 & 37.80 & 36.69 & 21.38 & 19.58 & 11.57 & 10.29 \\
\hline 11:00-12:00 & 2177.03 & 42.14 & 42.42 & 23.92 & 22.56 & 12.95 & 11.67 \\
\hline $12: 00-13: 00$ & 2104.73 & 39.05 & 39.14 & 21.97 & 20.81 & 11.89 & 10.81 \\
\hline $13: 00-14: 00$ & 1863.42 & 32.94 & 32.95 & 18.43 & 17.98 & 10.13 & 9.36 \\
\hline $14: 00-15: 00$ & 1953.59 & 36.20 & 35.90 & 20.26 & 19.17 & 10.91 & 10.05 \\
\hline $15: 00-16: 00$ & 1984.12 & 38.46 & 37.66 & 21.81 & 20.39 & 12.01 & 10.60 \\
\hline $16: 00-17: 00$ & 2174.47 & 44.67 & 44.03 & 25.14 & 23.80 & 13.96 & 12.50 \\
\hline 17:00-18:00 & 2521.20 & 52.77 & 52.87 & 29.37 & 29.35 & 16.37 & 15.00 \\
\hline $18: 00-19: 00$ & 2792.91 & 62.51 & 62.05 & 34.98 & 34.74 & 19.50 & 17.85 \\
\hline 19:00-20:00 & 2743.83 & 54.97 & 54.93 & 30.65 & 30.03 & 16.93 & 15.72 \\
\hline \multicolumn{2}{|l|}{ Total } & 490.46 & 488.62 & 275.24 & 264.49 & 151.41 & 137.66 \\
\hline \multicolumn{2}{|c|}{ Communication gain } & \multicolumn{2}{|c|}{1.84} & \multicolumn{2}{|c|}{10.75} & \multicolumn{2}{|c|}{13.75} \\
\hline \multicolumn{2}{|c|}{ Maximum per period } & 62.51 & 62.05 & 34.98 & 34.74 & 19.50 & 17.85 \\
\hline \multicolumn{2}{|c|}{ Maximum per station } & 18.31 & 16.99 & 12.86 & 13.35 & 9.57 & 8.14 \\
\hline
\end{tabular}

We give in (12) the objective function used in 2BH model.

$$
\begin{aligned}
F_{2 B H}= & \omega\left(\sum_{i=1}^{N} \sum_{k=1}^{M A_{i}-1} \sum_{p=k+1}^{M A_{i}} C 1(i, k, p)+\sum_{i=1}^{N-1} \sum_{\substack{j=i+1 \\
i, j \in \\
\text { same site }}}^{N} \sum_{k=1}^{M A_{i}} \sum_{p=1}^{M A_{j}} C 2(i, j, k, p)\right) \\
& +\sum_{\substack{\left(S_{i}, S_{j}\right) \\
i<j \in[1 . . N]}} \sum_{\substack{\left(f_{i, k}, f_{j, p}\right) \\
k \in\left[1 . . M A_{i}\right] \\
p \in\left[1 . . M A_{j}\right]}} B_{i, j,\left|f_{i, k}-f_{j, p}\right|}^{2 B H}
\end{aligned}
$$

Table 4 shows computational results of classical and dynamic traffic modeling on the real world problem $D$. To obtain this table, we generate two frequency plans using the GTS algorithm. The first is built on the basis of classical traffic modeling (12). The second is built on the basis of our dynamic traffic modeling (7). The performance of each frequency plan, in terms of lost traffic, is estimated for each period using PARCELL ${ }^{\odot}$ simulator. We present also at the lower part of the table: the total lost traffic (column Total), the communication gain (difference between classical and dynamic model), the maximum lost traffic for one time period (robustness criteria) and finally the maximum lost traffic for one cell. Information is given for each of the three quality thresholds $(2 \%, 4 \%$ and $7 \%)$. 
The experiments were carried out using the same parameters of the GTS algorithm: 100,000 generations for a population of 8 solutions with a crossover rate of 0.3 and Tabu search length of 30 . From Table 4, we notice that the dynamic model gives better frequency plans both in terms of global traffic capacity and robustness. Indeed, for the three thresholds, we get a gain of communication ranging from 1.84 and 13,75 Erlang.

\section{Conclusions}

This paper proposed an in-depth study for the channel assignment problem from both a modelling and solution point of view. The contribution of this paper is twofold. First, we proposed a dynamic traffic model for the fixed channel assignment problem. Contrary to classical models such as $2 \mathrm{BH}$ that consider the traffic as static data, the proposed model takes into account both temporal and spatial evolution of traffic, leading thus to a much more accurate and robust characterization of traffic. This dynamic model allowed us to introduce two new optimization criteria: frequency plan robustness and spatial repartition of interference, in addition to the classical interference criterion. The proposed model gives a new basis for developing more effective solution methods.

For the solution purpose, we developed a powerful Genetic Tabu search algorithm, which is mainly characterized by a problem specific crossover, an effective Tabu search mutation and a Tabu list shared by the individuals of the population. Extensive studies were carried out to study the behavior of this hybrid algorithm and the influence of its parameters. Based on fictitious and real data, experiments were realized to assess the performance of the genetic Tabu search algorithm and its underlying dynamic traffic model. Computational results show that using the proposed traffic model, the Genetic Tabu search algorithm gives better frequency plans in terms of the three quality criteria used. The gain of communications was also measured, showing a clear decrease of traffic lost. Finally, Genetic Tabu search was shown to dominate (unsurprisingly) the Tabu search algorithm alone and the hybrid algorithm with no shared Tabu list.

This proposed model introduces three optimization criteria. In this work, we used a simple penalty-based approach to deal with these criteria. An interesting alternative worth of investigation is the multi-criteria approach that is gaining recently much research efforts. Finally, the model itself may be further enriched to give different priority to periods (professional and private communication).

Acknowledgements We are grateful to the referees for their highly insightful comments and suggestions, which help us to improve the presentation of the paper. This work is partially supported by France Telecom R\&D (Orange), which is acknowledged.

\section{References}

1. Aardal, K.I., van Hoesel, C.P.M., Koster, A.M.C.A., Mannino, C., Sassano, A.: Models and solution techniques for the frequency assignment problem. J. 4OR 1(4), 261-317 (2003) 
2. Brélaz, D.: New methods to color vertices of graph. Commun. ACM 22, 251-256 (1979)

3. Chan, T.H., Palaniswani, M., Everitt, D.: Neural network-based dynamic channel assignment for cellular mobile communication systems. IEEE Trans. Veh. Technol. 43(2), 279-288 (1994)

4. Chang, K.-N., Kim, S.: Call blocking performance of new dynamic channel assignment scheme in cellular radio networks. In: Proc. of IEEE VTC, pp. 198-202 (1996)

5. Chen, L., Yoshida, S., Murata, H.: A dynamic channel assignment algorithm for voice and data integrated TDMA mobile radio. In: Proc. of IEEE VTC, pp. 213-217 (1996)

6. Colombo, G.: A genetic algorithm for frequency assignment with problem decomposition. Int. J. Mob. Netw. Des. Innov. 1(2), 102-112 (2006)

7. Crescenzi, P., Gambosi, G., Penna, P.: On-line algorithms for the channel assignment problem in cellular networks. Discrete Appl. Math. 137, 237-266 (2004)

8. Eisenblatter, A., Grotschel, M., Koster, A.: Frequency planning and ramifications of coloring. ZIBReport 00-47, December (2000)

9. Fischetti, M., Lepschy, C., Minerva, G., Romanin-Jacur, G., Toto, E.: Frequency assignment in mobile radio systems using branch-and-cut techniques. Eur. J. Oper. Res. 123, 241-255 (2000)

10. Fleurent, C., Ferland, J.A.: Genetic and hybrid algorithms for graph coloring. In: Laporte, G., Osman, I.H., Hammer, P.L. (eds.) Annals of Operations Research. Metaheuristics in Combinatorial Optimization, vol. 63 (1996)

11. Galinier, P., Hao, J.-K.: Hybrid evolutionary algorithms for graph coloring. J. Comb. Optim. 3(4), 379-397 (1999)

12. Gamst, A.: A resource allocation technique for FDMA systems. Alfa Freq. 57(2), 89-96 (1988)

13. Ghosh, S.C., Sinha, B.P., Das, N.: Channel assignment using genetic algorithm based on geometric symmetry. IEEE Trans. Veh. Technol. 52, 860-875 (2003)

14. Glover, F., Laguna, M.: Tabu Search. Kluwer Academic, Boston (1997)

15. Goldberg, D.E.: Genetic Algorithms in Search, Optimization, and Machine Learning. AddisonWesley, Reading (1989)

16. Hale, W.K.: Frequency assignment: theory and application. Proc. IEEE 68(12), 1498-1514 (1980)

17. Hao, J.K., Dorne, R., Galinier, P.: Tabu search for frequency assignment in mobile radio networks. J. Heuristics 4(1), 47-62 (1998)

18. Hellebrabdt, M., Lambrecht, F., Mathar, R., Niessen, T., Starke, R.: Frequency allocation and linear programming. In: Proc. of IEEE VTC, vol. 48, pp. 617-621 (1999)

19. Horng, J.-T., Jin, M.-H., Kao, C.-Y.: Solving fixed channel assignment problems by an evolutionary approach. In: Proc. of GECCO-2001, pp. 351-358 (2001)

20. Hurley, S., Whitaker, R.M., Smith, D.H.: Channel assignment in cellular networks without channel separation constraints. In: Proc. IEEE VTC, vol. 50, pp. 1714-1718 (2000)

21. IBM Corp. User Guide IBM Prospect 8.0. IBM, Raleigh (2009)

22. Jaimes-Romero, F.J., Muñoz-Rodrìguez, D.: Channel assignment in cellular systems using genetic algorithms. In: Proc. IEEE VTC, pp. 741-745 (1996)

23. Katzela, I., Naghshineh, M.: Channel assignment schemes for cellular mobile telecommunication systems: a comprehensive survey. IEEE Pers. Commun. (June), 10-31 (1996)

24. Lee, W.: Mobile Communications Design Fundamentals. Wiley Series in Telecommunications. Wiley, New York (1992)

25. Lee, Y., Kang, H.G.: Cell planning with capacity expansion in mobile communications: a Tabu Search Approach. IEEE Trans. Veh. Technol. 49(5), 1678-1691 (2000)

26. Lee, Z.J., Lee, C.Y.: A hybrid search algorithm with heuristics for resource allocation problem. Inf. Sci. 173, 155-167 (2005)

27. Leese, R., Hurley, S. (eds.): Methods and Algorithms for Radio Channel Assignment. Oxford Lecture Series in Mathematics and its Applications. Oxford University Press, Oxford (2002)

28. Luna, F., Alba, E., Nebro, A.J., Pedraza, S.: Evolutionary algorithms for real-world instances of the automatic frequency planning problem in GSM networks. In: Seventh European Conference on Evolutionary Computation in Combinatorial Optimization (EVOCOP 2007). Lecture Notes in Computer Science, vol. 4446, pp. 108-120. Springer, Berlin (2007)

29. Mac Donald, V.: Advanced Mobile Phone Service: The Cellular Concept. BELL Syst. Tech. J. (January), 15-41 (1979)

30. Mandal, S., Saha, D., Mahanti, A.: A real-time heuristic search technique for fixed channel allocation (FCA) in mobile cellular communications. Microprocess. Microsyst. 28, 411-416 (2004)

31. Matsui, S., Watanabe, I., Tokoro, K.: In: A Parameter-free Genetic Algorithm for a Fixed Channel Assignment Problem with Limited Bandwidth. LNCS, vol. 2439, pp. 789-799. Springer, Berlin (2002) 
32. Merz, P., Freisleben, B.: Fitness landscape analysis and memetic algorithms for the quadratic assignment problem. IEEE Trans. Evol. Comput. 4(4), 337-352 (2000)

33. Murray, K., Pesch, D.: Adaptive radio resource management for GSM/GPRS networks. In: First Joint IEI/IEE Symposium on Telecommunications Systems Research, 27 Nov. (2001)

34. Renaud, D., Caminada, A.: Evolutionary methods and operators for frequency assignment problem. SpeedUp J. 11(2), 27-32 (1997)

35. San José-Revuelta, L.M.: An heuristic search technique for fixed frequency assignment in nonhomogeneous demand systems. Signal Process. 88(6), 1461-1476 (2008)

36. Sastry, K., Goldberg, D.E.: Modeling tournament selection with replacement using apparent added noise. In: Proc. of the Genetic and Evolutionary Computation Conference (2001)

37. Taillard, A., Glover, F., De Werra, D.: A User's Guide to Tabu Search. Ann. Oper. Res. 41, 3-28 (1993)

38. Tajima, J., Imamura, K.: A strategy for flexible channel assignment in mobile communication systems. IEEE Trans. Veh. Technol. 37(2), 92-103 (1988)

39. Vasquez, M., Hao, J.-K.: A heuristic approach for antenna positioning in cellular networks. J. Heuristics 7(5), 443-472 (2001)

40. Wille, V., Multimaki, H., Irons, S.: A practical approach to channel borrowing for microcells in GSM systems. In: Proc. IEEE VTC, pp. 144-148 (1998)

41. Zander, J.: Radio resource management in future wireless networks: requirements and limitations. IEEE Commun. Mag. 30-36 (1997) 